\title{
Millimetre wave attenuation of prototype diagnostic components for the ITER bolometers
}

\author{
H. Meister ${ }^{\mathrm{a}}$, W. Kasparek ${ }^{\mathrm{b}}$, D. Zhang ${ }^{\mathrm{c}}$, M. Hirsch ${ }^{\mathrm{c}}$, J. Kolla ${ }^{\mathrm{a}}$, A. Zeitler ${ }^{\mathrm{b}}$ \\ ${ }^{a}$ Max-Planck-Institut für Plasmaphysik, Garching, Germany \\ ${ }^{b}$ Universität Stuttgart, Institut für Grenzflächenverfahrenstechnik $\mathcal{E}$ Plasmatechnologie, Stuttgart, Germany \\ ${ }^{c}$ Max-Planck-Institut für Plasmaphysik, Teilinstitut Greifswald, Greifswald, Germany
}

\begin{abstract}
Bolometers in current and future fusion devices, in particular those in ITER, are vulnerable to stray radiation from electron cyclotron resonance heating $(\mathrm{ECRH})$ which results in measurement errors for plasma radiation detection. To protect the detectors from this stray radiation in the millimetre wavelength range, dedicated diagnostic components have been designed and tested. One option is to place a top plate which contains a microwave-reflecting grid onto the collimators. Another option investigated is the coating of the collimator channels using a microwave absorbing ceramic. Measurements of the mm-wave attenuation of the collimator in front of the bolometer detectors with and without top plate or coated collimator channels have been performed in the frequency range of $125-420 \mathrm{GHz}$. The attenuation factor of the collimator channels at $170 \mathrm{GHz}$ (the ECRH frequency for ITER) with neither microwave grid nor coating is typically $10 \mathrm{~dB}$. The coating enhances this to $40 \mathrm{~dB}$ and including the microwave grid yields at least an attenuation factor of $70 \mathrm{~dB}$, which is sufficient to reduce the residual ECRH induced signal significantly below the one due to plasma radiation. Placing a bolometer camera (collimator connected to detector housing) inside the isotropic microwave field of the test facility MISTRAL, the attenuation factor of the full diagnostic set-up using a top plate was determined to be in the order of $45 \mathrm{~dB}$. This degraded attenuation implies that particular attention has to be paid to design and quality control of the joints of diagnostic components to prevent microwave leakage.
\end{abstract}

Keywords: ITER, bolometer, microwave attenuation, plasma diagnostics

\section{Introduction}

The total radiated power as well as the radiation emission profile on ITER will be determined by the bolometer diagnostic. A bolometer measures the plasma radiation over a wide spectral range (from soft- $X$ to the infrared) by monitoring the temperature rise induced by deposition of photon energy in the absorber layer of the bolometer. The reference detector type chosen for ITER is the metal resistor bolometer $[1,2]$. In order to determine the spatially resolved radiation profile, tomographic reconstruction routines are applied on the measurement results from many lines-of-sight (LOS). The LOS can be defined by collimators, which provide an individual aperture per detector pixel.

For measurements in ITER one has to take into account, that stray radiation from electron cyclotron resonance heating (ECRH) might occur in several of the foreseen operating scenarii. In case of non-optimal absorption isotropic stray radiation up to levels of
$90 \mathrm{~kW} / \mathrm{m}^{2}$ can be expected at the bolometer surfaces located behind the gaps between blanket modules of the first wall when assuming a gap size of $30 \mathrm{~mm}$ [3]. The propagation of $\mathrm{mm}$-waves from the entrance of the camera towards the detector is along metal surfaces with very high reflectivity. The latter also applies to the absorber. Thus, it can be assumed that an isotropic mmwave field is generated inside the camera because of multiple reflections. The power level corresponds to the power density at the collimator opening times its area and only a fraction, corresponding to the ratio of absorber surface to the other inner surfaces, leads to additional signals. Therefore, collimators already provide a basic attenuation of stray radiation. However, the levels of power from mm-waves may be higher than the expected levels of plasma radiation and thus lead to measurement errors if not further mitigated. In addition to the openings of the collimator channels, mm-waves may enter the camera housing also at joints if the connections of components are not microwave tight, as for a 
non-polarised wave there is always a polarisation angle which is parallel to the remaining slit and thus provides a path into the interior of the camera. Copper seals and overlapping edges are methods to reduce this effect.

In order to protect the detectors from stray radiation in the mm-wavelength range, several methods are known [4]: metal meshes, perforated metal plates and microwave absorbing coatings. In this work the latter two options have been used to manufacture prototype components of a bolometer camera suitable for ITER (section 2) and test them in the laboratory with respect to their ability to protect the detector from incident microwave radiation in the wavelength range between 125-450 GHz (section 3). Additionally, a complete setup of a bolometer camera was tested under isotropic microwave radiation at $140 \mathrm{GHz}$ in the test facility MISTRAL [5] (section 4). From the measurement results presented conclusions with respect to the application in ITER are drawn in section 5.

\section{Collimator prototypes}

The first version of the prototype collimator used for these investigations is shown on the left in figure 1. It consists of 4 viewing channels, which are split into 3 sub-collimators each in order to reduce the viewing cone perpendicular to the fan of LOS [6]. At the plasma facing side, a top plate is added, using overlapping edges of $5 \mathrm{~mm}$ length and a copper seal to prevent microwave leackage. Additionally, the top plate contains four microwave grids, one for each LOS. The cross-section of the sub-collimators are $1.23 \times 2.86 \mathrm{~mm}^{2}$. The microwave grids provide channels of $5 \mathrm{~mm}$ length with cross sections of $0.54 \times 0.6 \mathrm{~mm}^{2}$, which act as attenuating wave guides for the mm-waves. They are arranged in arrays of $6 \times 4$ channels with separating walls of $0.15 \mathrm{~mm}$ thickness completely filling out the opening surface of the collimators. This arrangement blocks about $50 \%$ of the opening surface for light transmission. The collimator body has been manufactured out of a solid block of TZM (molybdemnum alloy) using wire erosion. The top plate is also made of TZM.

The second version of the prototype collimator is shown in figure 1 on the right. Here, individual apertures for each LOS were placed inside two body parts to define the LOS. The division into sub-collimators was provided only by the apertures. The same top plate as in version 1 is placed on top of the collimator. Additionally, the two body parts were coated on the inside by a microwave absorbing ceramic $\left(\mathrm{Al}_{2} \mathrm{O}_{3} / \mathrm{TiO}_{2}\right)$ [7].

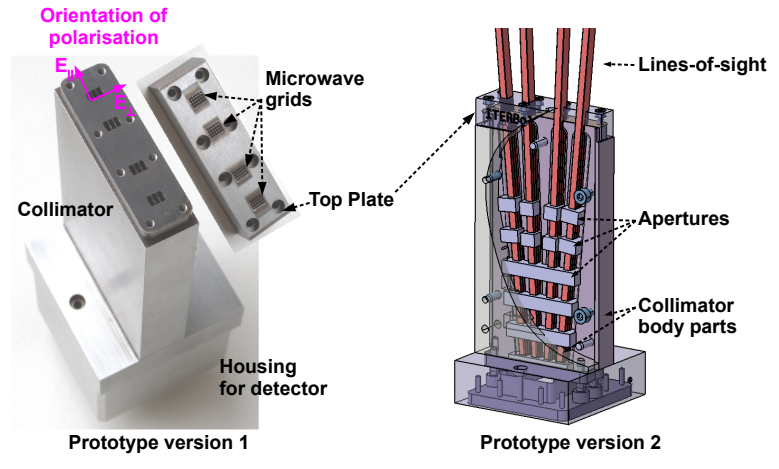

Figure 1: Collimator prototype versions used to determine the $\mathrm{mm}$ wave attenuation. Version 1 (photo on the left) is made out of a solid block of TZM. Version 2 (CAD drawing on the right) has been manufactured with individual apertures placed inside two body parts, which were coated by a microwave absorbing ceramic $\left(\mathrm{Al}_{2} \mathrm{O}_{3} / \mathrm{TiO}_{2}\right)$. The top plate with the perforated grid is identical for both.

\section{3. mm-wave attenuation in collimators}

To get information on the suppression of millimetre waves for the individual viewing channels, classical transmission measurements have been performed using the linear polarized fundamental TE10 mode. Measurements with the top plate installed as well as without the top plate were performed. The setup consisted of an ABmm Network-analyzer with a base frequency of 8-18 GHz, an active multiplier generating the 12th harmonic for the frequency range $125-225 \mathrm{GHz}$ and a transition from D-band to Ka-band waveguide which connected to one of the channels of the collimator (with or without top plate). The exit of the collimator, where the bolometer sensor is usually placed, used a transition from Ka-band to D-band waveguide which connected to a harmonic mixer. The active multiplier could also operate with less performance at the 24th harmonic, in the range of 225-450 GHz. For frequencies above $225 \mathrm{GHz}$ a narrowband detector was used. Here, measurements could only be done at single frequencies. The estimated accuracy of a single-frequency data point is typically $\pm 5 \mathrm{~dB}$. Measurements with the polarization parallel and perpendicular to the fan of LOS were done (see also figure 1). As reference, the transitions were connected directly. The attenuation of each channel is defined as the ratio between the transmission through the channel and the reference measurement.

Figure 2 shows the result of the attenuation measurements of the first version of the collimator. As can clearly be seen, the attenuation without a top plate is in the order of $10 \mathrm{~dB}$, with somewhat higher values measured for the parallel polarization due to the increased 

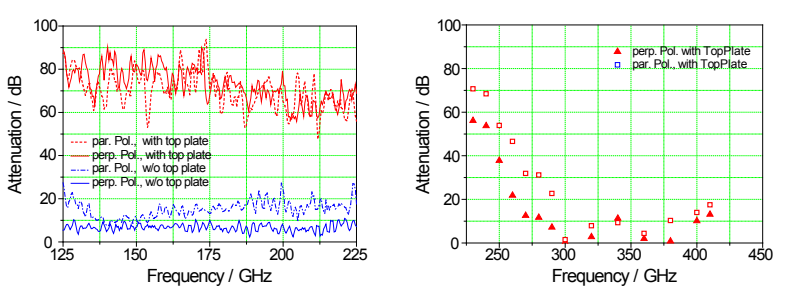

Figure 2: Attenuation measurements of the first collimator version without microwave coating, with (red) and without (blue) top plate. Polarisation parallel to the fan of LOS is indicated by broken lines and open symbols, perpendicular polarisation with full lines and full symbols.

matching loss at the entrance of the sub-channels. With the top plate mounted, the mm-wave attenuation by the collimator is at least $70 \mathrm{~dB}$, probably much higher. Here, the measured attenuation is limited by the dynamic range of the set up. Interference between coherent noise from the network analyzer and lowlevel crosstalk explains the noisy character of the traces. Essentially, the results have to be seen therefore as worst case of the attenuation of the collimators.

In the frequency range $225-410 \mathrm{GHz}$, the attenuation with the top plate mounted is shown in the right graph of figure 2. The attenuation is drastically decreased at frequencies above $250 \mathrm{GHz}$, where the apertures in the top plate become transparent (formal cut-off frequencies: $f_{c, \perp}=250 \mathrm{GHz}$, and $f_{c, \|}=278 \mathrm{GHz}$ ). At frequencies above $300 \mathrm{GHz}$, the typical suppression of stray radiation is below $10 \mathrm{~dB}$.

Figure 3 shows the result of the attenuation measurements of the second version of the collimator with a microwave coating. Compared to the first version with the uncoated channels, a strong increase of the attenuation is found. This can be seen in the measurements without the top plate mounted on the bolometer head, which are not influenced by limits in the dynamic range. At $170 \mathrm{GHz}$, the typical attenuation is $40-50 \mathrm{~dB}$, which is to be compared to about $10 \mathrm{~dB}$ for uncoated channels. An effect of the top plate is visible for $f<270 \mathrm{GHz}$. Above this frequency, the typical attenuation for the coated channels is $40 \mathrm{~dB}$. Note that the viewing channels become more and more overmoded with increasing frequency. This leads to mode conversion, standing waves etc., which causes strong fluctuations in the transmitted power. Therefore, the typical error in this data is of the order of $10 \mathrm{~dB}$.

To measure the transmission of isotropic radiation, the front side collimator was installed in a spherical untuned cavity (similar to sniffer probes, [8]). The output side of the collimator was connected to a second
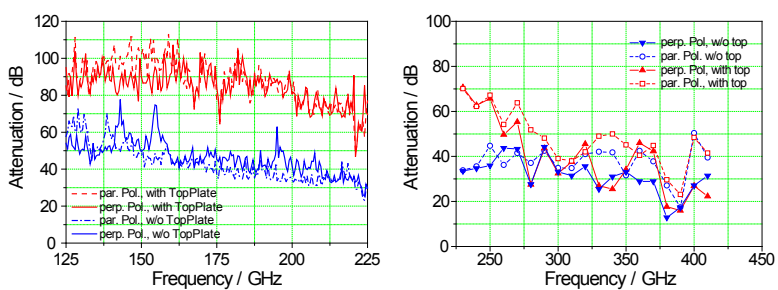

Figure 3: Attenuation measurements of the second collimator version with microwave coating, with (red) and without (blue) top plate. Polarisation parallel to the fan of LOS is indicated by broken lines and open symbols, perpendicular polarisation with full lines and full symbols.

untuned cavity. The input cavity was connected via a monomode hole to the generator as above, the output cavity to the harmonics mixer. For reference measurements, the two cavities were connected to each other via a hole with an area corresponding to the area of the bolometer mount at the rear side of the bolometer head. The power level measured in the output cavity for this situation defined $0 \mathrm{~dB}$ attenuation. The dynamic range for this set up was much lower than that for the transmission measurements. Nonetheless, within the given limits all results presented for parallel and perpendicular polarisation could be confirmed.

\section{4. mm-wave attenuation of a bolometer camera}

To test the mm-wave attenuation for a complete bolometer camera, the first version of the collimator has been mounted on a housing containing a bolometer detector and its signal connections. The joints of collimator and housing were designed with overlapping edges of $5 \mathrm{~mm}$ length and a copper seal to provide shielding against microwave leakage. This assembly was mounted on the end of an immersion tube which provided shielding for the signal cables when mounted in the test facility MISTRAL. This facility provides an isotropic microwave field of $760 \mathrm{~kW} / \mathrm{m}^{2}$ on all surfaces inside the vessel at a frequency of $140 \mathrm{GHz}$ [5]. The signal cables of the bolometer detector were connected to a typical bolometer data acquisition system being able to perform both, calibration and measurement as in [9].

For the tests, several microwave pulses between $5 \mathrm{~ms}$ and $100 \mathrm{~ms}$ duration have been injected into the vessel. Figure 4 shows typical results of the evaluated power as have been detected by the bolometer sensor during $100 \mathrm{~ms}$ ECRH pulses for various conditions. The light blue curve shows the power detected during a microwave pulse at $106^{\circ} \mathrm{C}$ operating temperature (after several conditioning pulses), the green one at $36^{\circ} \mathrm{C}$ 


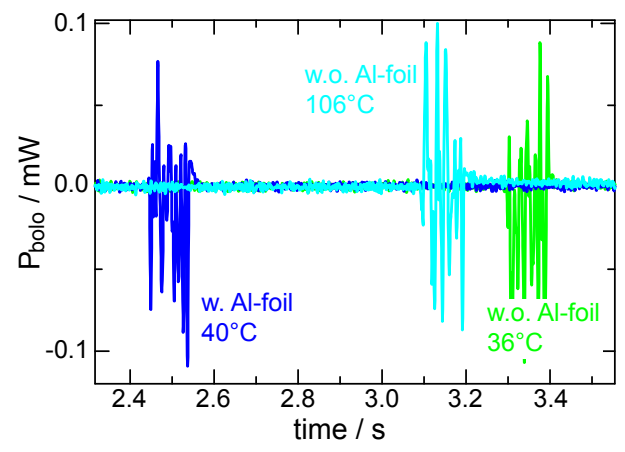

Figure 4: Detected power of the bolometer sensor during microwave pulses in MISTRAL at $36^{\circ} \mathrm{C}$ (green) and $106^{\circ} \mathrm{C}$ (light blue) operating temperature and a reference pulse with covered collimator channels at $40^{\circ} \mathrm{C}$ operating temperature (dark blue).

(without prior conditioning). A reference measurement was performed after covering the apertures of the collimator with aluminum tape to prevent the influx of microwaves from the top plate. As the corresponding dark blue curve demonstrates, this does not change the level of the detected power. This indicates that the temperature dependent calibration of the detectors did not lead to uncertainties and that a microwave leakage exists and is most probably located at the joint between either collimator and detector housing or the attached immersion tube. In all cases the detected power is in the order of $0.1 \mathrm{~mW}$, implying that the actual microwave power induced through the top plate is lower. The strong variations of the evaluated power during each microwave pulse are caused by frequency chirps of the gyrotrons during each pulse which lead to a mode structure. This in turn leads to variations of the microwave power depositions within the bolometer assembly. Thus, measurement and reference absorber detect varying amounts of microwave power during each pulse giving rise to both positive and negative signals.

With the area of each bolometer absorber of $6 \mathrm{~mm}^{2}$ and the power densitity provided by MISTRAL, the detected maximum power of $0.1 \mathrm{~mW}$ leads to the conclusion that the assembly as tested attenuates the incoming $\mathrm{mm}$-wave radiation by a factor of $4.56 \cdot 10^{4}$, or $47 \mathrm{~dB}$. This value can be regarded as a lower limit for the achieved attenuation as only the maximum absolute value of the measured power variations because of frequency chirps has been taken into account.

\section{Conclusion}

The attenuation of stray radiation from heating sources in ITER has been measured for two differ- ent bolometer collimators equipped with two different methods to attenuate incoming $\mathrm{mm}$-wave radiation. Depending on the methods applied, attenuation factors up to at least $70 \mathrm{~dB}$ have been obtained. Also, a complete bolometer camera assembly has been tested for microwave leakage in the test facility MISTRAL showing a reduced attenuation factor in the order of $45 \mathrm{~dB}$.

In view of the developments for ITER the attenuation factors observed for the collimator with a top plate including a microwave grid are able to reduce mmwave stray radiation to negligible values even for worst case assumptions if microwave leakage in other components can be prevented. Stray radiation levels of up to $90 \mathrm{~kW} / \mathrm{m}^{2}$ would be reduced to at most $10^{-2} \mathrm{~W} / \mathrm{m}^{2}$, which would not endanger the measurements of the lowest expected radiation levels in the order of $4 \mathrm{~W} / \mathrm{m}^{2}$ [10]. Using only a microwave coating, worst case assumptions lead to the expectation of ECRH stray radiation levels at the bolometer detector in the order of $9 \mathrm{~W} / \mathrm{m}^{2}$, which is comparable to the lowest expected radiation levels.

The measurements of the complete bolometer assembly in MISTRAL were performed using a coated collimator and a top plate with microwave grid. The resulting reduced attenuation of $47 \mathrm{~dB}$ demonstrates that microwave leakage occured at joints of components, in particular as a measurement with a completely covered top plate gave the same results. This points out that particular attention has to be paid to design and quality control of the joints of diagnostic components to prevent microwave leakage.

The results presented in this work provide thus a solid basis for deciding on the preferred method for attenuating ECRH stray radiation in the ITER bolometer diagnostic as soon as final information on their integration interfaces and on ECRH power levels are known.

\section{Acknowledgments}

This work was supported by funds from the German Ministry for Education and Research under the Grant No. 03FUS0006. The sole responsibility for the content presented lies with the authors.

\section{References}

[1] K. F. Mast et al. Rev. Sci. Instrum., 62(3):744-750, 1991.

[2] H. Meister et al. Rev. Sci. Instrum., 79:10F511, 2008.

[3] M. Clough et al. private communication, March 2013.

[4] D. Zhang et al. In Proc. of the 38 EPS Conference on Controlled Fusion and Plasma Physics, Strasbourg, 2011, volume 35G, Geneva, 2011. 
[5] S. Ullrich et al. 140-GHz test chamber for stray electron cyclotron radiation. Stellarator News, (98), May 2005.

[6] H. Meister and S. Kalvin. Fusion Eng. Des., 89(12):3039, 2014.

[7] Miriam Floristán et al. Fusion Eng. Des., 86(911):1847, 2011.

[8] F Gandini et al. Fusion Eng. Des., 56-57(0):975-979, 2001.

[9] L. Giannone et al. Rev. Sci. Instrum., 73(9):3205-3214, 2002.

[10] S Kalvin and G. Grunda. private communication, 2008. 